\title{
Influence of environmental features on the electric field
}

\author{
Yu Huang ${ }^{1,2,3}$, Xiaojuan Zhang ${ }^{1}$, Yanju $\mathrm{Gao}^{4}$ \\ 1. Guizhou hail prevention and control technology engineering center, Guiyang 550002 \\ 2. Key Laboratory for Cloud Physics of China Meteorological Administration,Beijing100000 \\ 3. Meteorological Disaster Defense Technology Center of GuiZhou Province, Guiyang 550002 \\ 4. Guiyang Yunan Lightning Protection Device Testing Station, Guiyang 550002
}

\begin{abstract}
In order to solve the influence of environmental factors on lightning electromagnetic field and improve the detection results, the distortion of electric field caused by environmental features, and given correction formula in the condition of electrostatic field, has been simulated. The results show that: in the condition of electrostatic field, on the ground,the higher the installation height, the greater the degree of electric field distortion. Electric field was shielded because of the existence of environmental features. When the electric field mill is on the top of the building, correction coefficient of electric field at building height varies significantly with the installation height, the building height is second, while it does not change obviously with building weight; The correction coefficient on the roof is bigger than on the ground when it is of the same the installation height. Besides that, the influence of surrounding buildings on electromagnetic radiation of lightning flash has been analyzed,the magnetic field has no obvious change to buildings exit or not,but the electric field also changes remarkably.
\end{abstract}

Keywords: Lightning Electromagnetic field; Finite difference method; Distortion; Shielding

\section{INTRODUCTION}

Electrostatic field observation in ground is an important part of the atmospheric electricity study, the deep research in the ground electric field becomes one of the important means of thunderstorms study. Atmospheric electric field monitoring measurement results are greatly influenced by the site environment in the ground. Buildings, trees, other projections and terrain can cause the significant change of atmospheric electric field nearby ${ }^{[1-2]}$. There are quite a few scholars ${ }^{[3-5]}$ think that goodness of fit in the inversion of charge structure by networking is too large because of site environment error. Finding out all kinds of environment's influence on the atmospheric electric field, giving different correction according to different environment has practical significance.

At present, the error revision of the instrument itself is usually based on the experiment ${ }^{[6-8]}$, while the impact of environment on atmospheric electric field is not clear, so it is unable to give some effective correction. We can find out the rule of atmospheric electric field affected by the environment using the combination of numerical simulation and actual detection. At the same time, it has been found that atmospheric electric field distortion degree increases with building height by placing an atmospheric electric field instrument in high-rise buildings for field observation ${ }^{[9-10]}$. Both the experiment and the simulation results of these research work is mainly in view of the low rise buildings, and is less considering the influence of tip or protrusions on the electric field distortion ${ }^{[11-12]}$. Yong-Bo Tan et.al ${ }^{[13]}$ found that atmospheric electric field distortion coefficient and cutting-edge height tends to linear increases relationship by numerical simulation. We concluded that the electric field instrument's own impact on atmospheric electric field is significant when it is on the roof. In simulation studies, most building shape is regular, and rarely consider the instrument's own influence on the top of the building, nor did correction, just think the detected results as the actual results of the influence of electric field.

We will use the finite difference method to research the distortion of electric field caused by site environmental under the background of homogeneous field before the lightning occurs and give correction formula. This paper is organized as follows. Section II presents the methodology for simulation. Section III simulate distortion of electric field caused by installation height and research environmental building's impact of electric field when the field mill is in the ground, taking the shielding effect into account, talk about influence of various factors on the detection results and give correction when the field mill is fixed on the top of the building. Further, shows the influence of surrounding buildings on electromagnetic radiation of lightning flash simply. Section IV concludes the paper and points out further work.

\section{METHODOLOGY}

The field mill widely used now mainly detected the vertical electric field, our research also focuses on it. In our study, the atmospheric electric field instrument itself and the environment is simplified to the basic unit of shape rules. Assumed that there is no free charge in model space, only a vertical downward electric field $E_{0}$ existed as background. The surface potential of ground and projections are set as zero.

In the absence of free charge, Poisson equation can be simplified into Laplace's equation ${ }^{[20]}$ :

$$
\nabla^{2} u=0
$$

In a two-dimensional model, Laplace's equation can be transferred to the equations in Cartesian coordinate:

$$
\frac{\nabla^{2} u}{x^{2}}+\frac{\nabla^{2} u}{y^{2}}=0
$$


The key to solve the problem is to find the solution of Poisson equation which satisfied the boundary conditions. As shown in figure 1, we divide grid in the light of $x=x_{i}$, $y=y_{j}$, in which $x_{i}=i h, y_{j}=j h, h=\frac{1}{N+1}, i, j=1,2, \ldots, N+1$, the grid points and the boundary point respectively set as:

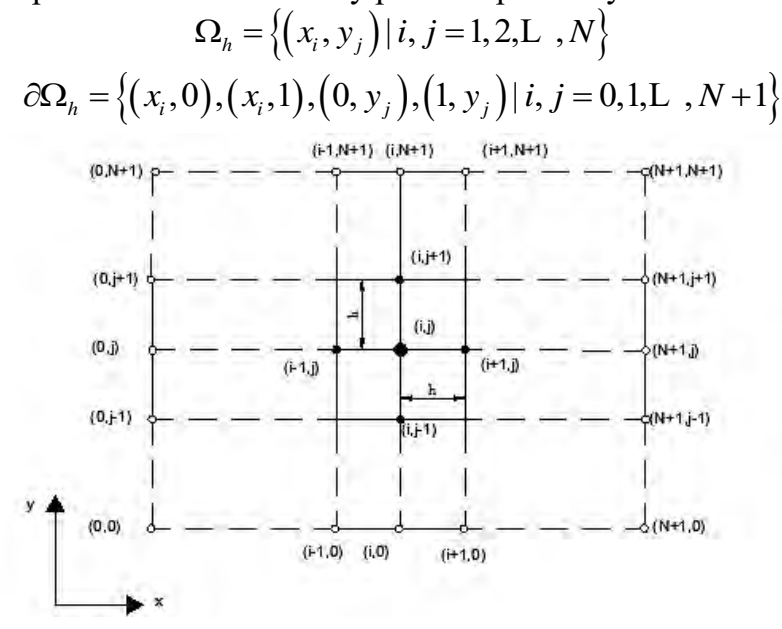

Fig 1. Difference schemes

The solve spacing in the graph is divided into $(N+1)^{2}$ square grid at the step $\mathrm{h}$, in which the unknown node counts to $N^{2}$. It formed a set of a linear equation with $N^{2}$ unknown quantities $u_{i j}, 1 \leq i, j \leq N$. We do Taylor series function to expansion $u(x, y)$, through transformation, the relationship between the five black points in Figure 1 is:

$$
\frac{1}{4}\left(\underline{u}_{i 1_{j}} \underline{u}{ }_{i 1}+u_{+i j}+u_{i j}\right)=u_{i j}
$$

The equation (3) can be solved combined with the given boundary condition (buildings, cutting-edge and ground potential is 0 , the air boundary meet Neumann boundary conditions, which means the potential gradient of the boundary is constant). We get the potential of each point in the selected area. To make the calculation speed accelerated, we adopts over relaxation iteration algorithm calculating the space potential $\mathrm{u}$. The electric field intensity $E$ on each point is equal to the negative gradient of potential $U^{[21]}$, we defined the ratio of electric field intensity $E$ at a grid point and $E_{0}$ as the electric field correction coefficient $\delta$ at the point.

\section{III . ANALYSIS AND RESULTS}

\section{A. The influence of instrument itself on electric field and}

\section{correction in ground}

We mainly discussed the impact of instrument installation height $h_{1}$ to electric field, which mostly revised by experiments at present. While we simulated and analyzed the influence on the electric field and gave correction by setting iteration grid detailed enough. Figure 2 shows the impact of $h_{1}$ to atmospheric electric field in ground in $10 \mathrm{~m} \mathrm{x} 10 \mathrm{~m}$ space, resolution of $10 \mathrm{~cm} \mathrm{x} 10 \mathrm{~cm}$, we can see that the electric field distortion degree increased with the increase of $h_{1}$ under the background of homogeneous field. According to the correction coefficient $\delta=E / E_{0}$ of different $h_{1}$, we get the correction fitting curve (figure 3 ).

The fixed expression of $\delta$ between $h_{1}$ can be described as $\delta=1.0586+2.6443 h_{1}-0.4485 h_{1}^{2}$. When we set $h_{1}$ as $1.5 \mathrm{~m}$, the correction coefficient is 3.9549 which accords with the experimental results in literature ${ }^{[7]}$.
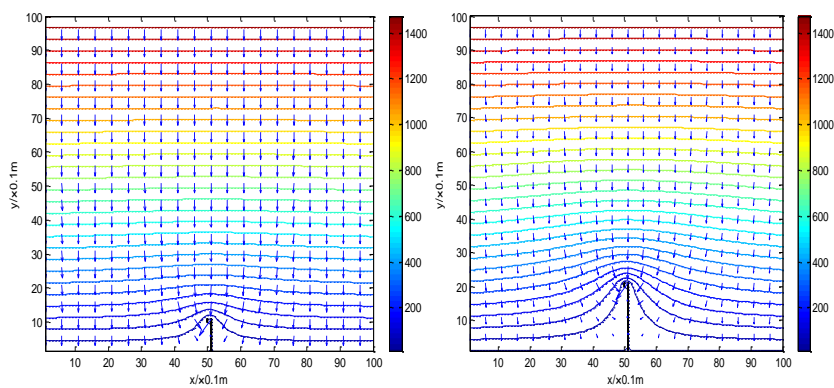

Fig 2. Impact of instrument installation height $h_{1}$ to the electric field distortion in ground (left $h_{1}=1 m$, right $h_{1}=2 m$ )

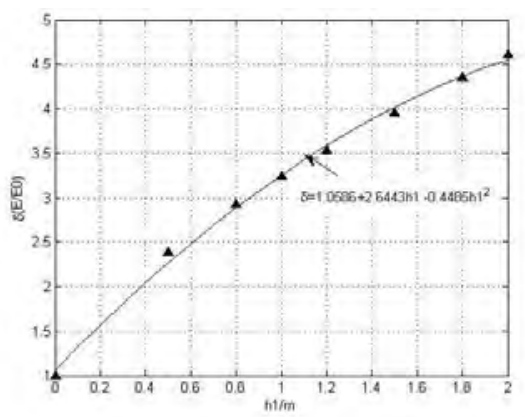

Fig 3. Correction relations and the fitting curve of $\delta=E / E_{0}$ to $h_{1}$

\section{B. Influence of environmental features on electric field} and correction within the shielding distance

The existence of construction makes the electric field distortion, produces a shielding effect on electric field in the ground. Previous work mainly analyzed the distortion coefficient of electric field(ratio of the electric field where the electric field distortion is the most serious and the background field), while we want to discuss the influence of surrounding buildings on the electric field and pay more attention to the change of electric field on the ground. Research results in literature ${ }^{[12]}$ show that the location of thunder cloud charge has no effect on the ratio of the electric field, and the higher the building the greater the electric field on the top of building within the horizontal distance of $15 \mathrm{~km}$, but it did not take the influence of building width into account. We calculated the electric field of a certain distance $D$ to the building, analyzed relationship between shielding distance $S$ with the building size, and discussed the relationship between $\delta\left(E_{2} / E_{0}\right)$ and $D$ by setting buildings height and width.

We can see from the table 1, shielding distance $S$ along with the height of the building is larger, as the change of width is smaller. Multiple relationship of shielding distance 
and the height of the building is between $1.83 \sim 3.2$, which is smaller than the literature ${ }^{[12]}$. It probably because we set the distance D from building border toward outside, while in the literature is building center outward, however the overall multiples range is similar.

Table I . Relationship between shielding distance S with $\mathrm{H}$ and $\mathrm{W}$

\begin{tabular}{|c|c|c|c|c|c|}
\hline \multirow{2}{*}{ Building width ( W ) } & \multicolumn{5}{|c|}{ Building height (H) } \\
\cline { 2 - 6 } & $10 \mathrm{~m}$ & $15 \mathrm{~m}$ & $20 \mathrm{~m}$ & $25 \mathrm{~m}$ & $30 \mathrm{~m}$ \\
\hline $5 \mathrm{~m}$ & 28 & 36 & 45 & 53 & 55 \\
\hline $10 \mathrm{~m}$ & 30 & 38 & 47 & 55 & 58 \\
\hline $15 \mathrm{~m}$ & 31 & 39 & 49 & 57 & 60 \\
\hline $20 \mathrm{~m}$ & 32 & 40 & 50 & 58 & 62 \\
\hline
\end{tabular}

Outside the shielding distance, the ground of the electric field is homogeneous field again, we discussed correction coefficient along with the change of parameter $\mathrm{D}$ within the shielding distance by building size $(\mathrm{H}=30 \mathrm{~m}, \mathrm{~W}=20 \mathrm{~m})$. Exponential relationship between $\delta$ with $\mathrm{D}$, several model is $\delta=a e^{\frac{b}{t}}$. Electric field at any point within the shielding distance can be calculated according to the model under the condition of the building size is known.

\section{Influence of various factors on the electric field and correction (Electric meter on the top of building)}

To avoid the impact of projections on the ground to electric field measurement, it has become popular to put the atmospheric electric field mill on the top of the building for measurement. Some researchers proposed to study the influence of the building height on electric field by comparing the electric field both on the roof and ground at the same time. While in the actual test, workers often make field test values directly as the real electric field at the top of the building, but it is ignored that instrument itself plays a significant role in the electric field distribution at the top of the building. As shown in figure 4 , the existence of electric meter at the top of building makes the electric field distribution become dense, so it is essential to study the impact of instrument itself to electric field at the top of the building and give electric correction at the building height. We set the $h_{1}=1 \mathrm{~m}$, mainly discuss the transformation of the electric field correction coefficient $\delta^{\prime}=E / E_{H}$ to the $\mathrm{H}, \mathrm{W}$ and $\mathrm{h} 1$ at the building height and put forward the correct relation between them.
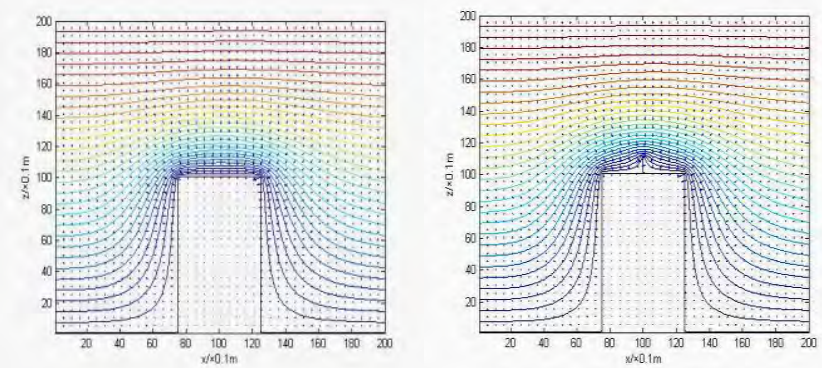

Fig 4. Potential distribution (left: without electric meter at the top of the building, with electric meter at the top of the building)

a. The change of the $\delta^{\prime}=E / E_{H}$ along with $H, W$

We set three groups of the width of the buildings, research $\delta$ ' along with the height of the building, from the results of figure 5 as you can see, the change tendency of $\delta$ ' in different width is consistent, and curves is more intertwined when $\mathrm{W}=$ $10,20, \delta^{\prime}$ tends to a fixed value when the width of building increase to a certain width. Combined with results showed in figure6, the change trend of $\delta$ ' with building width in different building height tends to straight line, which verifies the change of $\delta^{\prime}$ with the width is not so obvious. From figure 5,6 we can conclude that $\delta^{\prime}$ has a tendency to decrease when $\mathrm{H}$ increased to a certain degree, but the overall change interval guarantees between 3.5 - 3.7, in the actual process it can be averaged to 3.6 for correction.

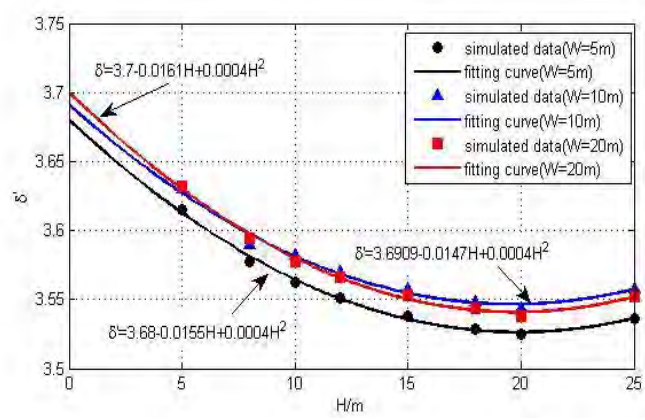

Fig 5. Change of $\delta^{\prime}$ along with the building height $\mathrm{H}$

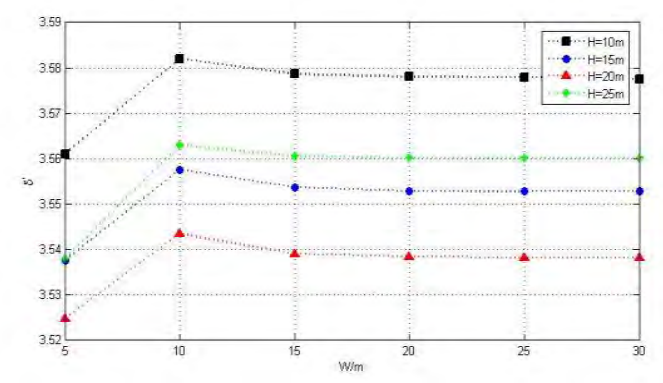

Fig 6. Change of $\delta^{\prime}$ along with the building width W

b. The change of the $\delta^{\prime}=E / E_{H}$ along with h1

When an electric field mill is put on the top of the building, not only the size of building but also the electric field 
instrument itself will impact the electric field on the top of building. We set up three groups of different sizes of buildings to research the electric field correction coefficient $\delta^{\prime}=E / E_{H}$ along with the change of electric field instrument installation height $h_{1}$ on the roof. From figure7 we can see that $\delta$ 'become bigger with the increase of $h_{1}$, but the rate of change is in the decrease. Compared with figure 3 , it is not hard to find that corresponding correction coefficient on the roof is bigger than on the ground of the same $h_{1}$, which is related to the electric field distortion degree on the roof. At the same time, curve trends are consistent under the condition of different construction size, while the difference in different height is bigger than in difference width, partly explains the change with the width is not obvious, and with height changes to a few bigger, but along with the change of $h_{1}$ is the largest.

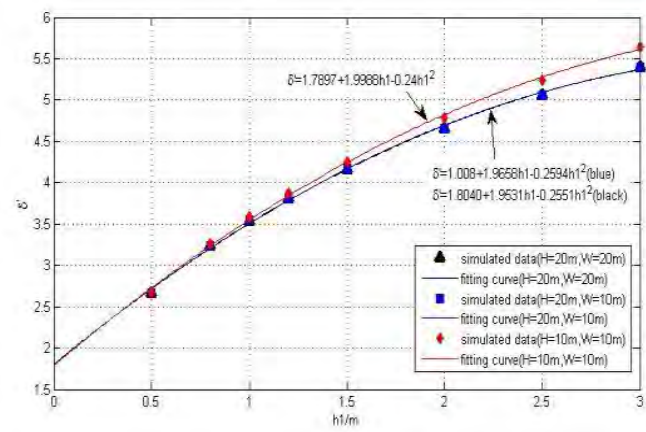

Fig7. Change of $\delta$ ' along with $h_{1}$

\section{Influence of surrounding buildings on electromagnetic radiation of lightning flash}

In order to study the influence of surrounding buildings on electromagnetic radiation of lightning flash, and thus improve the efficiency of lightning detection, established the integration space calculation model of buildings and lightning channel, based on the TL lightning counter current mode, using the finite difference time domain method made the numerical calculation of single and multiple buildings. the magnetic field has no obvious change to buildings exit or not, but the electric field changes remarkably.

The electric field before or behind the building were decreased because of existence of the building, the electric field in the middle area reduced more significantly when there are multiple buildings; the electric field at the top of the building was increased obviously, no matter single building or multiple buildings, and the proportion was in a stable range when the building height at a certain value.

\section{a. single building}

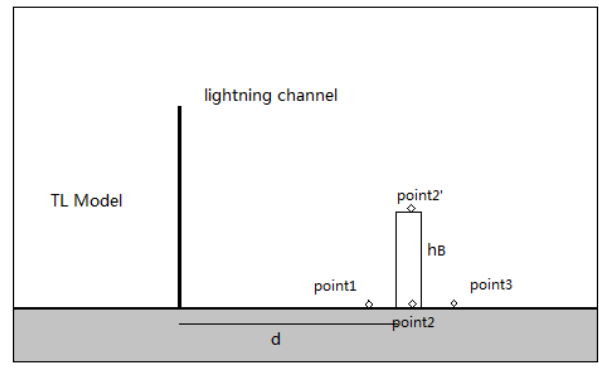

Fig9. single building model
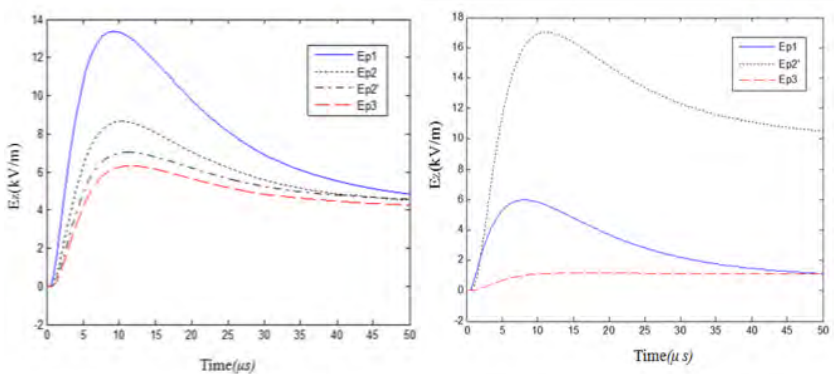

Fig10. Ez Without building(left),with building(right)

Table II Ratio of the Ez (with building/without building)at different point

\begin{tabular}{|c|c|c|c|c|}
\hline \multirow{3}{*}{ Point } & \multirow{2}{*}{$\mathrm{d}(\mathrm{m})$} & \multicolumn{3}{|c|}{$\mathrm{h}_{\mathrm{B}}, \mathrm{m}$} \\
\cline { 2 - 5 } & & 20 & 50 & 100 \\
\hline \multirow{3}{*}{ point 1} & 100 & 0.72 & 0.48 & 0.48 \\
\cline { 2 - 5 } & 200 & 0.71 & 0.40 & 0.24 \\
\cline { 2 - 5 } & 500 & 0.72 & 0.40 & 0.20 \\
\hline \multirow{3}{*}{ point ${ }^{\prime}$} & 100 & 1.12 & 1.44 & 2.43 \\
\cline { 2 - 5 } & 200 & 1.16 & 1.58 & 2.10 \\
\cline { 2 - 5 } & 500 & 1.22 & 1.60 & 2.06 \\
\hline \multirow{3}{*}{ po int 3 } & 100 & 0.72 & 0.33 & 0.27 \\
\cline { 2 - 5 } & 200 & 0.77 & 0.26 & 0.13 \\
\cline { 2 - 5 } & 500 & 0.79 & 0.14 & 0.09 \\
\hline
\end{tabular}

b. multiple buildings

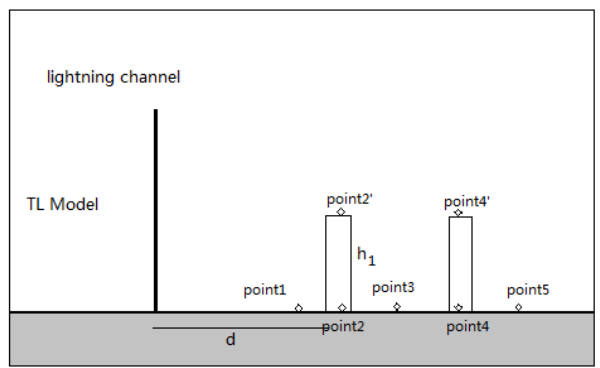

Fig11. multiple buildings model 
Table III Ratio of the Ez (with building/without building)at different point

\begin{tabular}{|c|c|c|c|c|}
\hline \multirow{2}{*}{ POINT } & \multirow[b]{2}{*}{$\mathrm{d}(\mathrm{m})$} & \multicolumn{3}{|c|}{ h1(m) } \\
\hline & & 20 & 50 & 100 \\
\hline \multirow{3}{*}{ point 1} & 100 & 0.71 & 0.43 & 0.30 \\
\hline & 200 & 0.68 & 0.36 & 0.22 \\
\hline & 500 & 0.72 & 0.38 & 0.19 \\
\hline \multirow{3}{*}{ point $2^{\prime}$} & 100 & 1.05 & 1.36 & 1.79 \\
\hline & 200 & 1.07 & 1.31 & 1.74 \\
\hline & 500 & 1.17 & 1.49 & 1.83 \\
\hline \multirow{3}{*}{ point 3} & 100 & 0.77 & 0.13 & 0.01 \\
\hline & 200 & 0.74 & 0.14 & 0.01 \\
\hline & 500 & 0.77 & 0.16 & 0.01 \\
\hline \multirow{3}{*}{ point $4^{\prime}$} & 100 & 1.16 & 1.43 & 1.75 \\
\hline & 200 & 1.17 & 1.27 & 1.52 \\
\hline & 500 & 1.22 & 1.49 & 1.78 \\
\hline \multirow{3}{*}{ point 5} & 100 & 0.95 & 0.22 & 0.06 \\
\hline & 200 & 0.87 & 0.17 & 0.03 \\
\hline & 500 & 0.77 & 0.17 & 0.03 \\
\hline
\end{tabular}

\section{SUMMARY}

In This paper, we have simulated, using the finite difference method in higher resolution, the distortion of electric field caused by instrument installation itself and environmental features under the background of homogeneous field before the lightning occurs, and given correction formula. Besides that, we analyzed the influence of surrounding buildings on electromagnetic radiation of lightning flash ,the magnetic field has no obvious change to buildings exit or not, but the electric field also changes remarkably.

The future research work will add experiment and multi-source detection data for more accurate analysis and get more accurate data, then explore the charge structure of thunderstorm .

\section{ACKNOWLEDGMENT}

This work was Supported by LCP/CMA (2017Z01603).

\section{REFERENCES}

[1]GOLDE R H,Lightning,1977,Academic Press Inc. (LONDON) L T D . [2]YANG Bo, ZHOU Bihua, GAO Taichang.Research on distribution of electric field near ground during thunderstorm process.Proc. Int. Sym p.on
EMC,Qingdao,China . 2007:267-260 .

[3] ZHANG Tinglong,QIE Xiushu,YUAN Tie, et al. The characteristics of cloud-to-ground lightning flashes and charge structure of a typical thunderstorm in Chinese inland plateau. Chinese Journal of Atmospheric Sciences (in Chinese), 2008,32(5): 1221- 1227.

[4] CUI Haihua, QIE Xiushu, ZHANG Qilin, et al. Equivalent Electrical Discharge Structure of Thunderstorm and Simultaneous Observation of Multi-Station of Cloud Flashes in Zhongchuan of Gansu. PLATEAU METEOROLOGY(in Chinese) , 2009,28(4): 808- 815.

[5] ZHANG Ting-long,YAN Muhong, ZHANG Tong, et al.Study of Charge Structure in a Thunderstorm Case of Zhongchuan Area by the Observation of Surface Electrical Field . PLATEAU METEOROLOGY (in Chinese), 2010, 29(6): 1524-1532.

[6]ZHANG Teng-fei,JI Xinyuan,XING Hongyan.Design of electric field calibration system, Meteorological, Hydrological and Instruments ( in Chinese),2012,4(12):67- 72.

[7]YANG Zhongjiang, Zhu Hao, TANG Hongke, et al. Research on source of error and analytical processing of atmospheric electric field data.

Transactions Atmospheric Sciences, 2010, 33 (6): 751-756.

[8]ZHOU Junchi . Calibration and Application of Data Measured by Electric Field Mill [D],2011.NUIST .

[9] Bermudez J. L, Rachidi F, Rubinstein M, et al. Far-field-current relationship based on the TL model for lightning return strokes to elevated strike objects. IEEE TRANSACTIONS ON ELECTROMAGNETIC COMPATIBILITY, Vol. 47, NO.1, pp: 146-159, 2005.

[10] Abbas M, Davide P, Farhad R, et al. Pierre Z 2007 7th International Symposium on Electromagnetic Compatibility and Electromagnetic Ecology Saint-Petersburg, Russia, 2007. June 26-29.

[11]Yoshihiro Baba, V. A. Rakov, Electromagnetic field at the top pf a tall building associated with nearby lightning return strokes [J]. IEEE TRANSACTIONS ON ELECTROMAGNETIC COMPATIBILITY , VOL. 49, NO. 3, pp: 632-643AUGUST 2007.

[12]GENG Xueying,ZHANG Qilin,LIU Mingyuan.Effect of Tall Buildings on Thunderstorm Electric Field. METEORLOGICAL SCIENCE AND TECHNOLOGY,2012,40 ( 5 ) :827- 833.

[13]GUO Xiufeng, TAN Yongbo, GUO Fengxia, et al. Numerical Simulation of Effects of Building Tips on Atmospheric Electric Field Distortion. Journal of application of meteorological , 2013,24(2) : 189- 196.

[14]HE wei, LIU Hongjian, Xu Lin, et al. Among the selective analysis lightning and protection lightning. Journal of Yunnan normal university, $2011,31(2): 9-11$.

[15]TANG Jun,XU Zhirong,CHEN Xiaoping, et al. Tall buildings of $10 \mathrm{kV}$ distribution line induction thunder lightning characteristics. The influence of electromagnetic arrester, 2012, 4:79- 84

[16] V. A. Rakov, "Lightning return stroke speed," J. Lightning Res., vol. 1, pp. 80-89, Jan. 2007.

[17]M. A. Uman.The Lightning Discharge . Orlando: Academic Press, 1987. $30-45$.

[18]Becerra M.,Cooray V.,Hartono Z.A.,Identification of lightning vulnerability points on complex grounded structures, Journal of Electrostatics, 2007. $65562-570$.

[19] LI Qing-yang,Wang Nengchao,YI Dayi.Numerical analysis [M].Tsinghua University Press .

[20] LV Yinghua, Numerical Method of Computational Electromagnetic[M]. Tsinghua University Press .

[21] GUO Shuohong, Electrodynamics [M]. Higher Education Press .

[22] YANG Yao, LIU Jie, CHEN Xu et al. Matlab used to Study Advanced Electric Field Characteristics and the Applications of the Conductor Near. Information and Communication, 2011.115 (5): 7-8. 